\title{
Nonlinear Scale invariant Formalism and its Application to Some Differential Equations
}

\author{
Anuja Ray Chaudhuri \\ Department of Mathematics, Ananda Chandra College \\ Jalpaiguri, West Bengal, Pin: 735101, India
}

\begin{abstract}
Scale free analysis has been developed recently and in this framework real number system is realized as an extended deformed real line $\mathrm{R}$. This analysis is applied on a class of ordinary differential equations. We report in particular some simple but nontrivial applications of this nonlinear formalism leading to emergence of complex nonlinear structures even from a linear differential system. These emergent nonlinear phenomena from a linear system is argued to offer, a new non-perturbative method for computing solutions and estimate amplitude, frequency etc. for a specific nonlinear system, viz. the Van der Pol equation.
\end{abstract}

Keywords: Scale invariance, non-archimedean absolute value, relative infinitesimals, nonlinear increment, non-perturbative method.

\section{Introduction}

World around us is nonlinear. The conventional treatments of nonlinear problems generally consider nonlinear (ordinry or partial) differential equations when actual nonlinearity appears as new terms with one (or more) (small) parameter(s), for instance, the pendulum equation, the Duffing equation, the Van der Pol oscillator [1]. The standard (regular) perturbation method attempts to find an approximate solution to a nonlinear problem, which can not be solved exactly, by starting from the exact solution of a related exactly solvable problem. But there are many limitations of perturbative methods. The standard approaches in resolving some of the limitations of the perturbation theory are the method of multiple time scales [2], homotopy analysis methods [3] and so on, besides the more sophisticated phase space(plane) analysis. Most of these methods rely and make use of some asymptotic matching of two or many branches of approximate solutions, obtained by solving some reduced component equations of the original equation, as the nonlinearity parameter asymptotically approaches some fixed value (may be $0, \infty$ or any fixed finite number).

With this rich background the present paper aims at formulating an altogether new approach in the study of nonlinear problems. In this paper we first present scale invariant formalism which is an extension of our previous work [4] and then apply this nonlinear analytic formalism to some differential equations starting from the very elementary level. In this formalism the real number system $\mathrm{R}$ is realized as an extended deformed real line $\mathrm{R}$ which reduces to the connected real line $\mathrm{R}$ only at the $\mathrm{O}(1)$ scale. At infinitesimally (infinitely) small (large) scales, $\mathrm{R}$ degenerates into a set having the structure of a positive measure totally disconnected Cantor set. In the presence of scale invariant infinitesimals, the usual point like structure of an element of the Cantor set is extended to an infinitesimal connected line segment (at the level of a well defined infinitesimal scale) on which a self similar replica of the original $\mathrm{O}(1)$ differential equation on $\mathrm{R}$ is written in a scale invariant manner using appropriate logarithmic variables. Two self similar replica equations on the disconnected branches are matched smoothly at the point of disconnection by inversion induced smooth jumps. We recall that such a smooth matching is not admissible in the ordinary analysis since a point of disconnection is interpreted as a singular point in the conventional treatment. The present nonlinear framework accommodating smooth jump as a nonlinear incremental mode is formulated mainly to bypass the difficulties in formulating differential equations on a disconnected setting involving discontinuous coefficients and/or data.

\section{Scale Invariant formalism}

The formulation of a scale invariant analysis was motivated by our effort in justifying the construction of the so-called nonsmooth solutions [5] of the simplest scale invariant Cauchy problem

$$
\mathrm{t} \frac{\mathrm{dX}}{\mathrm{dt}}=\mathrm{X}, \mathrm{X}(1)=1
$$

in a rigorous manner. It is clear that the framework of classical analysis, because of Picard's uniqueness theorem, can not rigorously accommodate such solutions, except possibly only in an approximate sense. To bypass the obstacle, it became imperative to look for a non-archimedean extension of the classical setting, thus allowing for existence of nontrivial infinitesimals (and hence, by inversion, infinities). Robinson's original 
models of nonstandard analysis (or any minor variations of the same) appeared to be unsatisfactory, because (i) infinitesimals here are infinitesimals even in "values", (ii) the value of an infinitesimal is the usual Euclidean value and (iii) these are new extraneous elements in R (a comparison with Nelson's approach [6] with the present one will be considered elsewhere). Although, the nonstandard $\mathrm{R}$ is non-archimedean, but still an infinitesimal behaves more in a "real number like" manner; that is to say, in essence, it fails to have an identity, except for its infinitesimal Euclidean value. Such nonstandard infinitesimals are known to generate proofs of harder theorems of mathematical analysis in a more intuitively appealling manner (see, for example, [7]). Further, any new theorem proved in the nonstandard approach is expected to have a classical analysis proof, though, may be, using lengthier arguments. Justifying a higher derivative discontinuous (nonsmooth) solution of (1), therefore, appeared to be difficult even in the conventional nonstandard analysis.

To counter this problem, we contemplated developing a novel non-archimedean extension of $\mathrm{R}$ by completing the rational number field $\mathrm{Q}$ under a novel ultrametric which treats arbitrarily small and large rational numbers seperately from finite, moderately large rational (and real) numbers [4]. The ultrametric reduces to the usual Euclidean value for finite real numbers, but, nevertheless, leads to a new definition (realization) of scale invariant infinitesimals in the present context. An important feature of this formalism is that we make use of traditional $\varepsilon-\delta$ techniques of classical analysis, but applied instead on a deformed real number system $\mathrm{R}$, when the deformation is induced by the ultrametric valuation of scale invariant relative infinitesimals (Definition 1,2).

We introduce scale invariant infinitesimals via a more refined evaluation of the limit $\mathrm{x} \rightarrow 0^{+}$in $\mathrm{R}$. Notice that as $\mathrm{x} \rightarrow 0^{+}$, there exists $\delta>0$ such that $0<\delta<\mathrm{x}$ and one usually identifies zero (0) with the closed interval $\mathrm{I}_{\delta}=[-\delta, \delta]$ at the scale (i.e. accuracy level in a computation) $\delta$. Ordinarily, $\mathrm{I}_{\delta}$ is a connected line segment, which shrinks to the singleton $\{0\}$ as $\delta \rightarrow 0^{+}$, so as to reproduce the infinitely accurate, exactly determinable ordinary real numbers. This also tells that in the usual (classical) sense there is no room for a scale when one talks about a limit of the form $\mathrm{x} \rightarrow 0$.

Let us now present yet another nontrivial mode realizing the limiting motion $\mathrm{x} \rightarrow 0^{+}$. This mode is nonlinear, as it is defined via an inversion law, rather than simply by linear translations, that is available uniquely to a real variable to undergo changes in the standard analysis on $\mathrm{R}$. In the presence of infinitesimals, the present nonlinear mode is shown to gain significance. In the following we give a definition and also present a nontrivial construction for an explicitly defined infinitesimal, without requiring the set up of Robinson's nonstandard analysis. As remarked already, our formalism offers a new, independent realization of inifinitesimals residing originally in an ultrametric space, but, nevertheless, inducing nontrivial influences in the form of an real valued "infinitesimal correction" of the form $\delta(\mathrm{x}) \propto \mathrm{x} \log \mathrm{x}^{-1}$ to an arbitrarily small real variable $\mathrm{X}$ approaching 0 , thus making the existence of infinitesimals analytically (and dynamically as well) more meaningful and significant. As a consequence, a real number $\mathrm{X}$, as it was, is raised to deformed values $\mathrm{X}_{ \pm} \in \mathrm{R}$ (Sec. 3), because of a possible nontrivial motion close to 0 . This construction also reveals the exact sense how a scale might become relevant in the present scale invariant formalism.

Definition 1. $[4,8,9,10,11]$ Let $\mathrm{x} \in \mathrm{I}=[-1,1] \subset \mathrm{R}$ and $\mathrm{x}$ be arbitrarily small, i.e., $\mathrm{x} \neq 0$, but, nevertheless, $|\mathrm{x}| \rightarrow 0^{+}$. Then there exists $\delta>0$ and a set of (positive) relative infinitesimals $\widetilde{\mathrm{x}}$ relative to the scale $\delta$ satisfying $0<\widetilde{\mathrm{x}}<\delta<\mathrm{x}$ and the inversion rule $\widetilde{\mathrm{x}} / \delta \propto \delta / \mathrm{x}$. The associated scale invariant infinitesimals are defined by $\widetilde{\mathrm{X}}=\lim _{\delta \rightarrow 0^{+}} \widetilde{\mathrm{x}} / \delta$.

Example 1. Let $\mathrm{x}_{\mathrm{n}}=\varepsilon^{\mathrm{n}(1-1)}, 0<1<1,0<\varepsilon<1$. Then scale invariant relative infinitesimals are $\widetilde{\mathrm{X}}_{\mathrm{n} \lambda}=\lambda \varepsilon^{\mathrm{nl}}, 0<\lambda<1$, when $\delta=\varepsilon^{\mathrm{n}}$, for a sufficiently large $\mathrm{n}$, is chosen as a scale. Analogously, for a continuous variable $\mathrm{x}$ approaching $0^{+}$, say, and considered as a scale, a class of the relative infinitesimals are represented as $\widetilde{\mathrm{x}}=\mathrm{x}^{1+1}(1+\mathrm{o}(\mathrm{x})), 0<1<1$, so that the corresponding scale invariant infinitesimals are defined by the asymptotic formula $\widetilde{X}=\lambda \mathrm{x}^{1}+\mathrm{o}\left(\mathrm{x}^{\mathrm{m}}\right), \mathrm{m}>1$. Notice that a scale invariant infinitesimal goes to zero at a smaller (ultrametric) rate $1: \tilde{X}=\lambda x^{1} \Rightarrow \frac{d \log \tilde{X}}{d \log x}=1$. 
Remark 1. In the limit $\delta \rightarrow 0$, the set of relative infinitesimals apparently reduces to the singleton $\{0\}$. However, the set of scale invariant infinitesimals is nevertheless nontrivial, in the sense that these will have nontrivial influences on the real number system. To uncover the nature of such influences, the set of infinitesimals, denoted $\mathbf{0}$, is now awarded with a natural weight (absolute value) with nontrivial effects on the number theory and other areas of analysis. The set $\mathbf{0}$ has the asymptotic representation $\mathbf{0}=\{0, \delta \widetilde{\mathrm{X}}\}$, as $\delta \rightarrow 0^{+}$. For definiteness, the ordinary zero (0) is called the stiff zero, when the nontrivial infinitesimals are called soft zeros. The ordinary real line $R$ is then extended over $\mathbf{R}=\{\widetilde{\mathbf{x}}: \widetilde{\mathbf{x}}=\mathrm{x}+\mathbf{0}, \mathrm{x} \in \mathrm{R}\}$, which as a field extension, and as a consequence of the Frobenius theorem, must be an infinite dimensional nonarchimedean space.

Definition 2. $[4,8,9,10,11]$ An absolute value $\mathrm{v}: \mathbf{0} \rightarrow \mathrm{I}^{+}=[0,1]$

$$
\mathrm{v}(\widetilde{\mathrm{x}}) \equiv\|\widetilde{\mathrm{x}}\|:=\lim _{\delta \rightarrow 0^{+}} \log _{\delta^{-1}} \widetilde{\mathrm{X}}^{-1}, \widetilde{\mathrm{x}}=\delta \widetilde{\mathrm{X}} \in \mathbf{0}
$$

together with $\mathrm{v}(0)=0$ is assigned to the set of infinitesimals $\mathbf{0}$. Infinitesimals weighted with above absolute value are called valued infinitesimals.

Remark 2. 1. As remarked already, the set of infinitesimals $0=\{0\}$ when $\delta \rightarrow 0$ classically. However, the corresponding asymptotic expressions for the scale free (invariant) infinitesimals are nontrivial, in the sense that the associated valuations (Definition 2) can be shown to exist as finite real numbers. Below we give a definite construction indicating the exact sense how relative infinitesimals and associated values arise in a limiting problem.

Fix a value of $\delta=\delta_{0}$ and let $\mathrm{C}_{\delta_{0}} \subset\left[0, \delta_{0}\right] \equiv \mathrm{I}_{\delta_{0}}^{+}$be a Cantor set defined by an IFS of the form

$$
\mathrm{f}_{1}(\mathrm{x})=\lambda \mathrm{x}, \mathrm{f}_{2}(\mathrm{x})=\lambda \mathrm{x}-\left(\lambda / \delta_{0}-1\right) \delta_{0}
$$

where $\lambda=\beta \delta_{0}, 0<\beta<1$ and $\alpha+2 \beta=1$. Thus, at the first iteration an open interval $\mathrm{O}_{11}$ of size $\alpha \delta_{0}$ is removed from the interval $\mathrm{I}_{\delta_{0}}^{+}$, at the second iteration 2 open intervals $\mathrm{O}_{21}$ and $\mathrm{O}_{22}$ each of size $\alpha \delta_{0}(\beta)$ are removed and so on, so that a family of gaps $\mathrm{O}_{\mathrm{ij}}$ of sizes $\alpha \delta_{0}(\beta)^{\mathrm{i}-1}, \mathrm{j}=1, \ldots, 2^{\mathrm{i}-1}$ are removed in subsequent iterations from each of the closed subintervals $\mathrm{I}_{\mathrm{ij}}, \mathrm{j}=1,2, \ldots, 2^{\mathrm{i}}$ of $\mathrm{I}_{\delta_{0}}^{+}$. Consequently, $\mathrm{C}_{\delta_{0}}=\mathrm{I}_{\delta_{0}}^{+}-\cup_{\mathrm{i}}^{\cup} \mathrm{O}_{\mathrm{ij}}=\overbrace{\mathrm{i}} \bigcup_{\mathrm{j}} \mathrm{I}_{\mathrm{ij}}$. Notice that the total length removed is $\sum \alpha \delta_{0}(2 \beta)^{\mathrm{i}-1}=\delta_{0}$, so that the linear Lebesgue measure $\mathrm{m}\left(\mathrm{C}_{\delta_{0}}\right)=0$.

Next, consider $\widetilde{\mathrm{I}}_{\mathrm{N}}=\left[0, \beta^{\mathrm{N}}\right]$ and let $\mathrm{N}=\mathrm{n}+\mathrm{r}$ and $\mathrm{N} \rightarrow \infty$ as $\mathrm{n} \rightarrow \infty$ for a fixed $\mathrm{r} \geq 0$. Choose the scale $\delta=\alpha \beta^{\mathrm{n}} \delta_{0}$ and define $\widetilde{\mathrm{x}}_{\mathrm{r}} \in\left[0, \alpha \beta^{\mathrm{N}} \delta_{0}\right]$ a ( positive ) relative infinitesimal (relative to the scale $\delta$ ) provided it also satisfies the inversion rule $\widetilde{\mathrm{x}} / \delta=\lambda \delta / \mathrm{x}$ (c.f. Definition 1), for a real constant $\lambda(\delta)$ $(0<<<1)$. For each choice of $\mathrm{x}$ and $\delta$, we have a unique $\widetilde{\mathrm{x}}$ for a given $\lambda \in(0,1)$. Consequently, by varying $\lambda$ in an open subinterval of $(0,1)$, we get an open interval of relative infinitesimals in the interval $(0, \delta)$, all of which are related to $\mathrm{x}$ by the inversion formula. In the limit $\delta \rightarrow 0$, relative infinitesimals $\widetilde{\mathrm{x}}_{\mathrm{r}}$, of course, vanish identically. However, the corresponding scale invariant infinitesimals $\widetilde{\mathrm{X}}_{\mathrm{r}}=\widetilde{\mathrm{x}}_{\mathrm{r}} / \delta, \delta \rightarrow 0$ are, nevertheless, nontrivial and weighted with new scale invariant absolute values via Definition 2.

The set of infinitesimals are uncountable, and as shown below the above norm satisfies the stronger triangle inequality $\mathrm{v}(\mathrm{x}+\mathrm{y}) \leq \max \{\mathrm{v}(\mathrm{x}), \mathrm{v}(\mathrm{y})\}$. Accordingly, the zero set $0=\left\{0, \pm \delta \widetilde{\mathrm{X}}_{\mathrm{r}} \mid \widetilde{\mathrm{X}}_{\mathrm{r}} \in\left(0, \beta^{\mathrm{r}}\right), \mathrm{r}=0,1,2, \ldots, \delta \rightarrow 0^{+}\right\}$may be said to acquire dynamically the structure of a Cantor like ultrametric space, for each $\beta \in(0,1 / 2)$ (so as to satisfy the open set condition [4]). The set 0 
indeed is realized as a set of nested circles $\mathrm{S}_{\mathrm{r}}:\left\{\widetilde{\mathrm{x}} \mid \mathrm{v}\left(\widetilde{\mathrm{x}}_{\mathrm{r}}\right)=\alpha_{\mathrm{r}}\right\}$, in the ultrametric norm, when we order, without any loss of generality, $\alpha_{0}>\alpha_{1}>\ldots$ The ordinary 0 of $\mathrm{R}$ is replaced by this set of scale free infinitesimals $0 \rightarrow 0^{\prime}=\mathbf{0} /^{\sim}=\left\{0, U_{r}\right\} ; \mathbf{0}^{\prime}$ being the equivalence class under the equivalence relation $\sim$, where $\mathrm{x} \sim \mathrm{y}$ means $\mathrm{v}(\mathrm{x})=\mathrm{v}(\mathrm{y})$. The existence of $\widetilde{\mathrm{x}}$ could also be concieved dynamically as a computational model $[4,8,9]$, in which a number, for instance, 0 is identified as an interval $[-\delta, \delta]$ at an accuracy level determined by $\delta=\beta^{\text {n }}$.

The concept of infinitesimals and the associated absolute value considered here become significant only in a limiting problem (or process), which is reflected in the explicit presence of " $\lim _{\delta \rightarrow 0}$ " in the relevant definitions. Recall that for the continuous real valued function $\mathrm{f}(\mathrm{x})=\mathrm{x}$, the statement $\lim _{\mathrm{x} \rightarrow 0} \mathrm{x}=0$, means that $\mathrm{x} \rightarrow 0$ essentially is $\mathrm{x}=0$. This may be considered to be a passive evaluation (interpretation) of limit. The present approach is active (dynamic), in the sense that it offers not only a more refined evaluation of the limit, but also provides a clue how one may induce new (nonlinear) structures (ingredients) in the limiting (asymptotic) process. The inversion rule (Definition 1) is one such nonlinear structure which may act nontrivially as one investigates more carefully the motion of a real variable $\mathrm{x}$ (and hence of the associated scale $\delta<\mathrm{x}$ ) as it goes to 0 more and more accurately. Notice that at any "instant", elements defined by inequalities $0<\widetilde{\mathrm{x}}<\delta<\mathrm{x}$ in a limiting process, are well defined; relative infinitesimals are meaningful only in that dynamic sense (classically, these are all zero, as $\mathrm{x}$ itself is zero). Scale invariant infinitesimals $\widetilde{\mathrm{X}}$, however, may or may not be zero classically. $\widetilde{\mathrm{X}}=\mu(\neq 0)$, a constant, for instance, is nonzero even when $\mathrm{x}$ and $\delta$ go to zero. On the other hand, $\widetilde{\mathrm{X}}=\delta^{\alpha}, 0<\alpha<1$, of course, vanish classically, but as shown below, are nontrivial in the present formalism. As a consequence, relative (and scale invariant) infinitesimals may said to exist even as real numbers in this dynamic sense. The acompaning metric $\|\cdot\|$, however, is an ultrametric.

However, a genuine (nontrivial) scale free infinitesimal $\widetilde{\mathrm{X}}$ can not be a constant. Let $\widetilde{\mathrm{x}}_{0}=\mu \delta, 0<\mu<1$, $\mu$ being a constant. Then $\mathrm{v}\left(\widetilde{\mathrm{x}}_{0}\right)=\lim _{\delta \rightarrow 0} \log _{\delta^{-1}} \mu=0$, so that $\widetilde{\mathrm{x}}_{0}$ is essentially the trivial infinitesimal 0 (more precisely, such a relative infinitesimal belongs to the equivalence class of 0 ).

The scale free infinitesimals of the form $\widetilde{\mathrm{X}}_{\mathrm{m}} \approx \delta^{\alpha} \mathrm{m}+\mathrm{o}\left(\delta^{\beta}\right), \beta>\alpha$ go to 0 at a slower rate compared to the linear motion of the scale $\delta$. The associated nontrivial absolute value $\mathrm{v}\left(\widetilde{\mathrm{x}}_{\mathrm{m}}\right)=\alpha_{\mathrm{m}}$ essentially quantifies this decelerated motion.

Theorem 1. $\mathrm{v}$ has following properties.

(i) $\mathrm{V}$ is an ultrametric, and hence $\mathbf{0}$ equipped with $\mathrm{V}$ is an ultrametric space.

(ii) $\mathrm{V}$ is a locally constant Cantor function.

Proof. (i) (a) $\mathrm{V}$ is well defined. Indeed, the open set 0 (in the usual topology, for each fixed $\delta$ ) is written as a countable union of disjoint open intervals $\mathrm{I}_{\delta \mathrm{i}}$ of relative infinitesimals $0=\bigcup_{\mathrm{I}_{\tilde{\mathrm{i}}}}$. Let $\mathrm{v}\left(\widetilde{\mathrm{x}}_{\mathrm{i}}\right)=\alpha_{\mathrm{i}}$, a constant for all $\widetilde{\mathrm{x}}_{\mathrm{i}}\left(=\lambda \delta \delta^{\alpha_{\mathrm{i}}}\right) \in \overline{\mathrm{I}}_{\delta \mathrm{i}}$, the closure of $\mathrm{I}_{\delta \mathrm{\delta}}$. Thus $\mathrm{v}$ exists and well defined.

(b) Let $0<\widetilde{\mathrm{x}}_{2}<\widetilde{\mathrm{x}}_{1}<\widetilde{\mathrm{x}}_{1}+\widetilde{\mathrm{x}}_{2}<\delta$ be two relative infinitesimals. We have, $0<\widetilde{\mathrm{X}}_{2}<\widetilde{\mathrm{X}}_{1}<\widetilde{\mathrm{X}}_{1}+\widetilde{\mathrm{X}}_{2}<1$ and $\quad \mathrm{v}\left(\widetilde{\mathrm{x}}_{2}\right)>\mathrm{v}\left(\widetilde{\mathrm{x}}_{1}\right)>\mathrm{v}\left(\widetilde{\mathrm{x}}_{1}+\widetilde{\mathrm{x}}_{2}\right)$, thus proving the strong triangle inequality $\mathrm{v}\left(\widetilde{\mathrm{x}}_{1}+\widetilde{\mathrm{x}}_{2}\right) \dagger \max \left\{\mathrm{v}\left(\widetilde{\mathrm{x}}_{1}\right), \mathrm{v}\left(\widetilde{\mathrm{x}}_{2}\right)\right\}$.

Next, given $0<\widetilde{\mathrm{x}}<\delta$, there exist a constant $0<\sigma(\delta)<1$ and a:0 $\rightarrow \mathrm{R}$, such that $\widetilde{\mathrm{X}}=\lambda \delta^{\mathrm{v}(\widetilde{\mathrm{x}})}$ and $\mathrm{v}(\widetilde{\mathrm{x}})=\sigma^{\mathrm{a}(\widetilde{\mathrm{x}})}$. Accordingly, $\mathrm{a}(\widetilde{\mathrm{x}}) \quad$ is a discrete valuation satisfying (i) $\mathrm{a}\left(\widetilde{\mathrm{x}}_{1} \widetilde{\mathrm{x}}_{2}\right)=\mathrm{a}\left(\widetilde{\mathrm{x}}_{1}\right)+\mathrm{a}\left(\widetilde{\mathrm{x}}_{2}\right)$, (ii) $\mathrm{a}\left(\widetilde{\mathrm{x}}_{1}+\widetilde{\mathrm{x}}_{2}\right) \geq \min \left\{\mathrm{a}\left(\widetilde{\mathrm{x}}_{1}\right), \mathrm{a}\left(\widetilde{\mathrm{x}}_{2}\right)\right\}$. As a result, $\mathrm{v}\left(\widetilde{\mathrm{x}}_{1} \widetilde{\mathrm{x}}_{2}\right)=\mathrm{v}\left(\widetilde{\mathrm{x}}_{1}\right) \mathrm{v}\left(\widetilde{\mathrm{x}}_{2}\right)$. Hence, $\{0, v\}$ is an ultrametric space. 
(ii) Let $0=\left(\cup \overline{\mathrm{I}}_{\delta \mathrm{i}}\right) \bigcup\left(\cup \mathrm{J}_{\mathrm{k}}\right)$, the closure of 0 . The open intervals $\mathrm{J}_{\mathrm{k}}$ are gaps between two consecutive closed intervals $\overline{\mathrm{I}}_{\delta \mathrm{1}}$. $\mathrm{J}_{\mathrm{k}}$ 's actually contain new points those arise as the limit points of sequences of the end points of the open intervals $I_{\delta i}$. Clearly, 0 is connected in usual topology. However, in the ultrametric topology, both $I_{\delta \mathfrak{1}}$ and $J_{k}$ are clopen sets and 0 is totally disconnected. Since, it is bounded and also is perfect 0 is equivalent to an ultrametric Cantor set.

Now, the local constancy of $\mathrm{v}$ in the ultrametric 0 follows from the definition:

$$
\frac{\mathrm{dv}(\widetilde{\mathrm{x}})}{\mathrm{dx}}=\lim _{\delta \rightarrow 0^{+}} \frac{\mathrm{d}}{\mathrm{dx}}\left(\frac{\log \mathrm{x}}{\log \delta}+1\right)=0
$$

The vanishing derivative above arises from a logarithmic divergence arising from the nontrivial finer scales. This is unlike the ordinary analysis, when one interprets 0 as a connected subset of $\mathrm{R}$, thereby forcing $\mathrm{V}$ to vanish uniquely, so as to recover the usual structure of $\mathrm{R}$. The above vanishing derivative can be interpreted nontrivially as a LCF [10] when $\mathrm{x} \in \mathrm{R}$ is supposed to belong to a Cantor subset of $\mathrm{I}$.

$\mathrm{Eq}(4)$ also reveals the reparaterization invariance of a locally constant valuation $\mathrm{v}(\mathrm{x})$. As a consequence, $\mathrm{V}$ may be a function of any reparametrized monotonic variable $\widetilde{\mathrm{x}}=\widetilde{\mathrm{x}}(\mathrm{x})$ with $\widetilde{\mathrm{x}}^{\prime}(\mathrm{x})>0$, instead being simply a function of the original real variable $\mathrm{X}$.

Now to construct a general class of locally constant functions in the ultrametric space, let us proceed as in (ia) above, with the supposition that the constants $\alpha_{\mathrm{i}}$ 's are arranged in ascending order. Thus, $\mathrm{v}\left(\widetilde{\mathrm{x}}_{\mathrm{i}}\right)=\alpha_{\mathrm{i}}, \alpha_{\mathrm{i}} \leq \alpha_{\mathrm{j}} \Leftrightarrow \mathrm{i} \leq \mathrm{j}$ for all $\widetilde{\mathrm{x}}_{\mathrm{i}} \in \mathrm{I}_{\mathrm{i}}$ (we drop the suffix $\delta$ for simplicity). Clearly, (4) holds over for all $I_{i}$. On the other hand, for an $\widetilde{x} \in J_{k}$, where $J_{k}$ separates two consecutive $I_{i}$ and $I_{i+1}$, say, so that $\widetilde{\mathrm{x}}_{\mathrm{i}}<\widetilde{\mathrm{x}}<\widetilde{\mathrm{x}}_{\mathrm{i}+1}$, where $\widetilde{\mathrm{x}}_{\mathrm{i}}$ is the right end point of $\mathrm{I}_{\mathrm{i}}$ and $\widetilde{\mathrm{x}}_{\mathrm{i}+1}$ is the left endpoint of $\mathrm{I}_{\mathrm{i}+1}$, we have $\mathrm{v}\left(\widetilde{\mathrm{x}}_{\mathrm{i}+1}\right)-\mathrm{v}\left(\widetilde{\mathrm{x}}_{\mathrm{i}}\right)=\left(\alpha_{\mathrm{i}+1}-\alpha_{\mathrm{i}}\right)$. Because of ultrametricity, one can always choose $\alpha_{\mathrm{i}}=\beta_{\mathrm{ij}_{\mathrm{i}}} \sigma(\mathrm{i})^{\mathrm{s}}$, for $\beta_{\mathrm{ij}_{\mathrm{i}}}>0$ ascending and $\sigma(\mathrm{i}) \rightarrow 0$ as $\mathrm{i} \rightarrow \infty$ and $\mathrm{j}_{\mathrm{i}}=0,1,2, \ldots \mathrm{k}(\mathrm{i})$ for some $\mathrm{i}$ dependent constant $\mathrm{k}(\mathrm{i})$ (c.f. 2nd para of (1b) ). Consequently, $v\left(\widetilde{\mathrm{x}}_{\mathrm{i}+1}\right)-\mathrm{v}\left(\widetilde{\mathrm{x}}_{\mathrm{i}}\right)=\left(\beta_{(\mathrm{i}+1) \mathrm{j}_{i+1}}-\beta_{\mathrm{ij}_{\mathrm{i}}}\right) \sigma(\mathrm{i})^{\mathrm{s}}$. It follows that the sequence $\mathrm{v}\left(\widetilde{\mathrm{x}}_{\mathrm{i}+1}\right)$ is increasing and $\mathrm{v}\left(\widetilde{\mathrm{x}}_{\mathrm{i}}\right)$ is decreasing. Thus, $\mathrm{v}(\widetilde{\mathrm{x}}):=\lim \mathrm{v}\left(\widetilde{\mathrm{x}}_{\mathrm{i}}\right)$ as $\mathrm{i} \rightarrow \infty$. Hence, $\mathrm{v}: 0 \rightarrow \mathrm{I}^{+}$is indeed a Cantor function.

Conversely, given a Cantor function $\phi(\mathrm{x}), \mathrm{x} \in \mathrm{I}^{+}$, one can define a class of infinitesimals $\widetilde{\mathrm{x}} \approx \delta \delta^{\phi(\widetilde{\mathrm{x}} / \delta)}$ belonging to the extended set 0 for $\delta \rightarrow 0^{+}$. This completes the proof.

Remark 3. To re-emphasise, the valuation $\mathrm{v}$ can be considered to quantify the degree of decelerated motion as the real variable $\mathrm{x} \rightarrow 0$ because of obstructions offered by nontrivial scale invariant infinitesimals in the ultrametric Cantor set of $\mathbf{0}$. The usual Euclidean norm is a measure of a finite real number because of its position relative to 0 .

Definition 3. Besides the usual Euclidean value, a real variable $\mathrm{x} \neq 0$, but $\mathrm{x} \rightarrow 0^{+}$gets a deformed ultrametric value given by $\mathrm{v}(\mathrm{x}):=\lim _{\delta \rightarrow 0^{+}} \log _{\delta^{-1}}(\mathrm{x} / \delta)$. Then $\mathrm{v}(\mathrm{x})=\mathrm{v}(\widetilde{\mathrm{x}})$.

Proof. Because of inversion rule, $\mathrm{x} / \delta=\lambda(\delta / \widetilde{\mathrm{x}}), 0<\lambda<1$, and hence $\mathrm{v}(\mathrm{x})=\mathrm{v}(\widetilde{\mathrm{x}})$ since $\lim \log _{\delta^{-1}} \lambda^{-1}=0$.

To proceed further, let us formulate some basic features of $\mathrm{v}$ revealing its nature of variability.

Lemma 1. Let $0<|\mathrm{x}|<\left|\mathrm{x}^{\prime}\right|$ be two arbitrarily small real variables and $\delta$ be a scale such that $0<\delta<\left|\mathrm{x}-\mathrm{x}^{\prime}\right|<|\mathrm{x}|<\left|\mathrm{x}^{\prime}\right|$. Then $\mathrm{v}\left(\mathrm{x}^{\prime}\right)=\mathrm{v}(\mathrm{x})$.

Proof. From definition $3, \mathrm{v}\left(\mathrm{x}-\mathrm{x}^{\prime}\right)<\mathrm{v}(\mathrm{x})<\mathrm{v}\left(\mathrm{x}^{\prime}\right)$. But $\mathrm{x}^{\prime}=\mathrm{x}+\left(\mathrm{x}^{\prime}-\mathrm{x}\right)$. So by ultrametric inequality, $\mathrm{v}\left(\mathrm{x}^{\prime}\right) \leq \max \left\{\mathrm{v}(\mathrm{x}), \mathrm{v}\left(\mathrm{x}^{\prime}-\mathrm{x}\right)\right\} \leq \mathrm{v}(\mathrm{x})$. 
Lemma 2 Let $0<|\mathrm{x}|<\left|\mathrm{x}^{\prime}\right|$ be two arbitrarily small real variables and $\delta$ and $\delta^{\prime}$ be two scales such that $0<\delta<|\mathrm{x}|<\delta^{\prime}<\left|\mathrm{x}^{\prime}\right|$. The corresponding scale invariant infinitesimals are $\widetilde{\mathrm{X}}$ and $\widetilde{\mathrm{X}}^{\prime}$ with associated valuations $\mathrm{v}(\mathrm{x})$ and $\mathrm{v}\left(\mathrm{x}^{\prime}\right)$. Then $\mathrm{v}\left(\mathrm{x}^{\prime}\right)=(\alpha / \mathrm{s}) \mathrm{v}(\mathrm{x})$, where $\alpha=\lim \log _{\widetilde{\mathrm{X}}} \widetilde{\mathrm{X}}^{\prime}$, determines the gap size between $\widetilde{\mathrm{X}}$ and $\widetilde{\mathrm{X}}^{\prime}$ and $\mathrm{s}=\lim \frac{\log \delta}{\log \delta}$ is the Hausdorff dimension of the Cantor set of infinitesimals as $\mathrm{x}, \mathrm{x}^{\prime} \rightarrow 0$

Proof. The proof follows from

$$
\frac{\mathrm{v}\left(\mathrm{x}^{\prime}\right)}{\mathrm{v}(\mathrm{x})}=\lim \frac{\log \left(\mathrm{x}^{\prime} / \delta^{\prime}\right)}{\log (\mathrm{x} / \delta)} \times \lim \frac{\log \delta}{\log \delta^{\prime}}
$$

so that $\alpha=\lim \log _{\mathrm{x} / \delta}\left(\mathrm{x}^{\prime} / \delta\right)=\lim \log _{\widetilde{\mathrm{X}}} \widetilde{\mathrm{X}}^{\prime} \Rightarrow \widetilde{\mathrm{X}}^{\prime}=\mathrm{X}^{\alpha}\left(1+\mathrm{O}\left(\beta\left(\mathrm{x}, \mathrm{x}^{\prime}\right)\right), \beta \rightarrow 0\right.$ faster than the linear approach $\mathrm{x} \rightarrow 0$.

Corollary 1. Let $0<\delta<\delta^{\prime}<\mathrm{x}$ be two scales in association with an arbitrarily small real variable and $\widetilde{\mathrm{X}}=(\mathrm{x} / \delta)^{-1}$ and $\tilde{\mathrm{X}}^{\prime}=\left(\mathrm{x} / \delta^{\prime}\right)^{-1}$ be the corresponding scale invariant infinitesimals. Then $\mathrm{v}\left(\mathrm{x}^{\prime}\right)=(\alpha / \mathrm{s}) \mathrm{v}(\mathrm{x})$, where $\alpha=\lim \log _{\tilde{\mathrm{X}}} \tilde{\mathrm{X}}^{\prime}$, determines the gap size between $\tilde{\mathrm{X}}$ and $\tilde{\mathrm{X}}^{\prime}$ and $\mathrm{s}=\lim \frac{\log \delta}{\log \delta^{\prime}}$ is the Hausdorff dimension of the Cantor set of infinitesimals as $\mathrm{x}, \mathrm{x}^{\prime} \rightarrow 0$.

Definition 4. A scale invariant jump is defined by the pure inversion $\tilde{X}^{\prime}=X^{-\alpha}$ with the scale invariant minimal jump size $\alpha=1$. The (scale invariant) jump size $\alpha$ thus runs over the set of natural numbers $\mathrm{N}$.

Remark 4. Lemma 1 characterizes the equivalence classes of infinitesimals with identical valuations. Subsequent lemma (and corollary) tells that the valuation $\mathrm{V}$ changes only when an infinitesimal from one equivalence class switches over to another class.

Summing up the above observations, we now state a general representation of relative infinitesimals and corresponding valuation.

Lemma 3. [10] A relative infinitesimal $\widetilde{\mathrm{x}}$ relative to the scale $\delta$ has the dominant asymptotic form

$$
\widetilde{\mathrm{x}}=\delta \times \delta^{1} \times \delta^{\phi(\widetilde{\mathrm{x}} / \delta)}(1+\mathrm{o}(1))
$$

with associated valuation $\mathrm{v}(\widetilde{\mathrm{x}})=1+\phi(\widetilde{\mathrm{x}} / \delta)$, where $1 \geq 0$ is a constant and $\phi$ is a nontrivial Cantor function.

Proof. The locally constant $\mathrm{v}=\mathrm{v}_{0}+\mathrm{v}_{1}$ solves $\frac{\mathrm{dv}}{\mathrm{dx}}=0$ and so the above ansatz is the more general solution, with the trivial ultrametric valuation $\mathrm{v}_{0}=1$ and the nontrivial valuation $\mathrm{v}_{1}=\phi$. The representation for $\widetilde{\mathrm{x}}$ now follows from definition.

Remark 5 As a real variable $\mathrm{x}$ and the associated scale $\delta<\mathrm{x}$ approach 0 , the corresponding infinitesimals $0<\widetilde{\mathrm{x}}<\delta$ may live (in contrast to measure zero Cantor sets considered so far) in a positive measure Cantor set $\mathrm{C}_{\mathrm{p}}$, say. Such a possibility is already considered in [10] in relation to an interesting phenomenon of growth of measure. In such a case $v_{0}(\widetilde{x})=m\left(C_{p}\right)=1$, the Lebesgue measure of $C_{p}$. The nontrivial component $v_{1}$ then relates to the uncertainty (fatness) exponent of the positive measure 1-set. In this extended model, the valuation quantifies the presence of nontrivial motion in a limiting process: $\mathrm{v}_{0}$ gives the uniform scale invariant motion when $\mathrm{v}_{1}$ arises from the associated nonuniformity stemming out from measure zero Cantor sets. 
Definition 5 The norm $\|\cdot\|$, induced by $v$, in $\mathbf{R}$ is defined by $\|\mathbf{x}\|=x, 0 \neq x \in R$, but $\|x\|=v(x)$, when $\mathrm{x} \in \mathbf{0}$.

To summarize, the ordinary real number system $\mathrm{R}$ is extended to a nonarchimedean space $\mathrm{R}$ with nontrivial valued infinitesimals. More details of the structure of $\mathrm{R}$ is available in [4]. These valued infinitesimals now, in turn, leave an imprint on the real number set so as to deform the original set [11]. As become evident the deformed system will be archimedean with the usual topology.

\section{Deformed Real Number System}

An ordinary real $\mathrm{X}$ is extended over to the fattened variables $\mathrm{X}_{ \pm}=\mathrm{x}_{ \pm}, \mathrm{X}_{ \pm}=\mathrm{X}^{\mp \mathrm{v}(\widetilde{\mathrm{x}} / \mathrm{x})}$ (so that $\mathrm{X}_{+}>\mathrm{x}$ and $\mathrm{X}_{-}<\mathrm{X}$ ). The fattened variables $\mathrm{X}_{ \pm}$live in a space $\mathrm{R}$ called the deformed real number set. Clearly, $\mathrm{R} \subset \mathrm{R}$, since $\mathrm{v}(0)=0$. The deformation is induced by scale invariant infinitesimals $\widetilde{\mathrm{X}}$ living in the ultrametric space $0 /\{0\}$. Recall that, scale invariant elements of the extended ultrametric space $\mathbf{R}$ in an infinitesimally small neighbourhood of $\mathrm{x}$ belongs to an ultrametric Cantor set $\mathrm{C}$ (homeomorphically equivalent to a $Z_{\mathrm{p}}$ ) and undergo changes by inversions of the form $\widetilde{\mathrm{X}}_{ \pm}=\widetilde{\mathrm{X}}_{ \pm} / \mathrm{x} \rightarrow \widetilde{\mathrm{X}}_{ \pm}{ }^{\prime}=\widetilde{\mathrm{X}}_{ \pm}^{ \pm \mathrm{e}^{\mathrm{h}}}, \mathrm{h} \in 0$ [10]. Such ultrametric infinitesimals and the associated inversions and related properties in $\mathrm{R}$, in turn, leave an imprint on the deformed set $R$, so that the real valued deformation factors $X_{ \pm}$not only live in an associated Cantor set (in usual topology) $\mathrm{C} \subset \mathrm{R}$, in the deformed neighbourhood of 1 , but also can be thought to undergo changes by inversions of the form $\mathrm{X}_{ \pm} \rightarrow \mathrm{X}_{ \pm}{ }^{\prime}=\mathrm{X}_{ \pm}^{ \pm \mathrm{e}^{\mathrm{h}}}$ where $\mathrm{h}$ now is a real parameter. Notice that there exists a natural homeomorphism between $\mathrm{C}$ and $\mathrm{C} \subset \mathrm{R}$. As a consequence, $\mathrm{R}$ locally has the structure of a positive measure Cantor set. Notice that given a real number $\mathrm{x}$ and a scale $\delta$, there exists a continuous mapping $\mathrm{f}: \mathrm{R} \rightarrow \mathrm{R}$ such that $\mathrm{x}=\delta \mathrm{f}(\delta)$. In the deformed system, this mapping $\mathrm{f}$ is induced by ultrametric infinitesimals in the form $\mathrm{f}(\delta)=\delta^{-\mathrm{v}(\widetilde{\mathrm{x}})}$.

Now, let us recall that differential shift increments in $\mathrm{R}$ are defined by $\mathrm{x} \rightarrow \mathrm{x}^{\prime}=\mathrm{x}+\mathrm{h}$ so that $\mathrm{dx}=\mathrm{x}^{\prime}-\mathrm{x}=\mathrm{h}, \mathrm{h} \rightarrow 0$. In the present case, the inversion induced jump increments in $\mathrm{R}$, over and above the usual shift differential, are defined by $d_{j} x=\log \log _{X} X^{\prime}=\log \log \left(\widetilde{x}_{0} \widetilde{x}\right.$ ) (we drop the suffix \pm for simplicity), where $X^{\prime}>X>1$, say. Indeed, for each $X$ which can be interpreted as a scale ( $\left.>1\right)$, there exists a class of 'infinitesimals' (actually, 'infinities') $X$ ' lying in a connected interval so that $\log _{X} X$ ' tends to a nonzero constant ( $>1$, say) $\alpha$ as the real variable $\mathrm{x} \rightarrow 0$. As a consequence, a line segment acquires a fractal like structure: a singleton $\{\mathrm{x}\}$ of the real number system $\mathrm{R}$, under the scale invariant action of the said infinitesimals, now gets deformed and fractured into a Cantor like set. The scale invariant extension of the line segment inhabiting the variable $\mathrm{X}$ is the Cantor set $\mathrm{C} \subset \mathrm{R}$ and $\alpha=\log _{\widetilde{\mathrm{X}}} \widetilde{\mathrm{X}}^{\prime}$ gives an estimate of the size of a jump connecting two points $X$ and $X^{\prime}$ in $C$ (c.f. Lemma 2). Clearly, the above estimate can also be written as $\log \left(\widetilde{\mathrm{X}}_{0} / \widetilde{\mathrm{X}}\right)$, where a point $\widetilde{\mathrm{X}}_{0}$ of a Cantor set is replaced by a connected segment over which the real variable $\widetilde{\mathrm{x}}$ (with a slight abuse of notation, we are here using the same symbol which denoted infinitesimals in $0)$ is supposed to live in $[8,9]$.

In the light of the above remarks, the following two lemmas now succintly encode the various incremental modes in the deformed space $R$.

Lemma 4. The scale invariant deformed variables $X$ living in a neighbourhood of 1 in the Cantor like subsets of $\mathrm{R} / \mathrm{R}$ undergo transitions by inversions $\mathrm{X} \rightarrow \mathrm{X}^{-\alpha}$, where $\alpha>0$ is a multiple of a natural number.

Lemma 5. Let $X \in R$ and $X \in R$. The differential increments for $X \in R$ are classified as: (i) $X^{\prime}=X+h$ for linear shifts taking over $R$, (ii) $X^{\prime}=e^{h^{\prime}} X \Rightarrow \log X^{\prime}=\log X+h^{\prime}$, for infinitesimal scaling and (iii) $\mathrm{X}^{\prime}=\mathrm{X}^{\mathrm{e}^{\mathrm{h}}} \Rightarrow \log \log \mathrm{X}^{\prime}=\log \log \mathrm{X}^{-1}+\mathrm{h}^{\prime \prime}$, for nonlinear inversions on $\mathrm{R} / \mathrm{R}$. As a consequence, the 
linear and nonlinear increments are related as $\mathrm{h}^{\prime}=\log \mathrm{h}$ and $\mathrm{h}^{\prime \prime}=\log \log \mathrm{h}$, where $\mathrm{h}, \mathrm{h}^{\prime}$ and $\mathrm{h}^{\prime \prime}$ are sufficiently small real variables.

These two lemmas are almost self explanatory in view of the above remarks and Lemma 2. The infinitesimal scaling is an effect of non-zero infinitesimals belonging to a specific equivalence class, having a nonzero constant valuation $\mathrm{v}(\widetilde{\mathrm{x}})=\alpha$, since $\mathrm{X}=\mathrm{x} \cdot \delta^{\mathrm{v}(\widetilde{\mathrm{x}})}=\mathrm{x} \cdot \delta^{\alpha}$, over a connected gap. Nontrivial inversion induced variations are revealed only under double logarithmic scales of an ordianry linear variable, when there is a transition from one gap to another (that is to say, between two points of the underlying Cantor set, for more details see $[9,10])$.

Theorem 2. The Cantor function $\mathrm{v}(\widetilde{\mathrm{x}})$ solves (4) everywhere in $\mathrm{I}^{+}$, thereby inducing a smoothening of the ordinary derivative discontinuity at a point $\widetilde{\mathrm{x}}_{0}$ of the underlying Cantor set $\mathrm{C}$, say. The actual variability of $\mathrm{v}$ is reflected at a logarithmic scale $\log \left(\widetilde{\mathrm{x}}_{0} / \widetilde{\mathrm{x}}\right)$ in the deformed space $R$, i.e.,

$$
\log \mathrm{y} \frac{\mathrm{dv}}{\mathrm{d} \log \mathrm{y}}=-\mathrm{v}, \mathrm{y}=\widetilde{\mathrm{x}}_{0} / \widetilde{\mathrm{x}}
$$

when the $\widetilde{\mathrm{x}}_{0} \in \mathrm{C}$ is replaced by an infinitesimal connected line segment in which the variable $\widetilde{\mathrm{x}}$ lives.

Proof. The Cantor function $\mathrm{v}: \mathrm{I} \rightarrow \mathrm{I}$ is constant on the gaps of the Cantor set $\mathrm{C}$. At a point of the Cantor set, the variation of $\mathrm{V}$ is given by Lemma 2. It follows from the fattened variable representation that $\mathrm{V}$ solves (7) thus establishing the variability of $v$ in the $\log$ arithmic variables, viz, $d \log v=-d \log \log \left(x_{0} / \widetilde{x}\right)$, in the neighbourhood of each $\mathrm{x}$ close to 0 when both $\mathrm{X}$ and 0 are supposed to be embedded in $\mathrm{R}$.

So far we have discussed about the scale free analysis. Next we study some ordinary differential equations in the light of this nonlinear formalsm.

\section{Ordinary Differential Equations}

\subsection{First order Equation}

Let us consider the simplest differential equation

$$
\frac{\mathrm{dx}}{\mathrm{dt}}=1(8)
$$

This may be assumed to represent the uniform motion (of the centre of mass) of a rigid ball. Accordingly, the ball will roll at uniform rate 1 along the $\mathrm{X}$-axis when the position at any instance may, for instance, be given by $\mathrm{x}(\mathrm{t})=\mathrm{t}$. This is what follows according to Newton's first law of motion. The ball will continue to roll for ever along the rectilinear path unless impressed by an externally applied force.

Now suppose the above uniform motion of the rigid ball continues to hold good even as $t \rightarrow \infty$. Let $\mathrm{t}=\frac{1}{\delta} \tau, \delta>0$. Then for $\delta \rightarrow 0^{+}, \mathrm{t} \rightarrow \infty$ for a finite $\mathrm{O}(1)$ non-zero $\tau$. In the new rescaled variable $\tau$, $\mathrm{Eq}(8)$ assumes the form of an singularly perturbed problem

$$
\delta \frac{\mathrm{dx}}{\mathrm{d} \tau}=1
$$

For an arbitrarily small but fixed $\delta \neq 0$, both the above equations are identical and yield the same solution $\mathrm{x}(\mathrm{t})=\mathrm{t}=\frac{1}{\delta} \tau$ (and also satisfies the initial condition $\left.\mathrm{x}(0)=0\right)$. It is also clear that the parameter $\delta$ can go arbitrarily close to 0 , but can not exactly vanish. In other words, the singularity at $\infty$ for $\mathrm{Eq}(8)$ is realized as a singularity at 0 for $\mathrm{Eq}(9)$. Consequently, this singular problem is further reducible to the scale free equation

$$
\tau \frac{\mathrm{dx}}{\mathrm{d} \tau}=\mathrm{x}
$$

when $\tau$ here is a small scale variable and tends to 0 satisfying : $0<\delta<\tau, \tau \rightarrow 0$. This follows once one replaces $\delta$ in the left hand side by $\tau / \mathrm{x}$, since $\mathrm{x}=\mathrm{t}$ for any finite value of $\mathrm{t}$.

Because of scale invariance, the above equation (10) is assumed to be valid on the deleted set (neighborhood) $\mathrm{I}=(-1,1) \backslash\{0\}$. Since, $\tau=0$ is unattainable, and the size of the hole of I has no positive lower bound, it 
might be imagined to have the shape of a totally disconnected Cantor set having a countable number of disjoint gaps (open intervals) of arbitrarily small lengths. Indeed, exploiting scale invariance, $\mathrm{Eq}(10)$ may be rewritten as

$$
\tau_{1} \frac{\mathrm{dx}}{\mathrm{d} \tau_{1}}=\mathrm{x}
$$

where $\tau_{1}=\tau / \delta$ and $0<\delta<\tau$ so that as $\tau \rightarrow 0^{+}, \delta \rightarrow 0^{+}$in such a manner that $\tau_{1}=\delta^{-v(\widetilde{\tau}(\tau))}$ goes to zero at a much slower rate. Here, $\mathrm{v}$ denotes an ultra metric valuation defined over the class of infinitesimals $\tilde{\tau}(\tau)$ those are assumed to reside in $(0, \delta)$. We remark here once more that the scale $\delta$ may be identified with an accuracy level in the sense that beyond which elements of $(0, \delta)$, are practically invisible (undetectable) relative to the real variable (defined by) $\tau>\delta$. Let $\mathrm{T}:=\log _{\delta^{-1}} \tau_{1}=\mathrm{v}(\widetilde{\tau})$. We call $\mathrm{T}$ as a dressed (deformed) value for the original linear variable (asymptotic time) $\tau$ relative to the scale $\delta$. Exactly in a similar manner we also write $\mathrm{X}:=\log _{\delta^{-1}} \mathrm{X}_{1}$ where $\mathrm{x}_{1}=\mathrm{x} / \delta$.

We now have an important observation. The limit $\delta \rightarrow 0^{+}$realizes a non classical extension of the ordinary linear neighborhood of 0 of the form $(-\delta, \delta)$ into a topologically inequivalent ultra metric neighborhood $\widetilde{U}$ so that the singleton set $\{0\}$ of $\mathrm{R}$ is extended into a positive measure Cantor like fractal set $\widetilde{\mathrm{C}} \subset \mathrm{R}$ (more precisely, the gaps of $\widetilde{\mathrm{C}}$ ) accommodating infinitesimals $\widetilde{\tau}$. The valuation $\mathrm{v}(\widetilde{\tau})$ now replaces the ordinary linear measure $\tau$ for the closed interval $[0, \tau]$ by the nonlinear, but, nevertheless, smooth measure $\mathrm{dT}=\operatorname{dv}(\widetilde{\tau})$ defined instead over an infinitesimal neighborhood (in the form of open gaps) of $\widetilde{\mathrm{C}} \subset(-\delta, \delta)$, as $\delta \rightarrow 0^{+}$. Clearly, the original initial value (consistency constraint) $\dot{\mathrm{x}}(0)=1$ for (8) gets extended smoothly over to a differential equation over $\widetilde{\mathrm{C}}$ in the form

$\frac{\mathrm{dX}}{\mathrm{dT}}=1$

using the deformed variables $\mathrm{X}$ and $\mathrm{T}$, thereby replicating the original ODE (8) on the deleted neighborhood $(-1,1) \backslash\{0\}$ onto an infinitesimally small (deleted) neighborhood $\widetilde{\mathrm{C}}$ of 0 . Accordingly, the original $\mathrm{t}-\mathrm{X}$ plane in the neighborhood of 0 is extended over to a $\mathrm{T}-\mathrm{X}$ plane (realized as a subset of the product space $\widetilde{\mathrm{C}} \times \widetilde{\mathrm{C}}$ ), which is nothing but the $\mathrm{t}-\mathrm{x}$ plane in the log-log scale, though however, in the asymptotic limit $\delta \rightarrow 0$.

To summarize, the extension of the singleton set of the form $\{0\}$ of $\mathrm{R}$ over to a deleted set of the form $(-1,1) \backslash\{0\}$ is realized explicitly in the context of the linear equation (8). Plugging in all the steps together we can also write down a generalized class of solutions of this equation in the form

$$
\mathrm{X}(\mathrm{t})=\mathrm{t}\left(1+\delta \times(\delta \mathrm{t} \uparrow)^{-\mathrm{X}(\mathrm{v}(\widetilde{\tau})}\right)=\mathrm{t}\left(1+\delta \times \tau^{-\mathrm{s}(\tau)}\right)
$$

where symbols are already introduced above. We remark that $t$ in the right hand sides of the new solution is an undirected real variable, but $\mathrm{t} \uparrow$ within the bracketed expression in the r.h.s (first equality) is an asymptotically increasing variable. For arbitrarily small $\delta>0$ and $\mathrm{t}: \mathrm{O}(1)$, the new extended solution reduces to the standard solution $\mathrm{x}(\mathrm{t})=\mathrm{t}$. However, as $\mathrm{t}$ grows to the level of $\mathrm{t} \sim \mathrm{O}\left(\delta^{-1}\right)$ so that $\tau \sim \mathrm{O}(1)$, the second terms in the two equalities can become significant, defining not only the above mentioned extension of the size of the ordinary neighborhoods of a point, but should also have important applications in various nonlinear complex physical, biological and other problems.

It also follows that the ordinary non-directed (classical/Newtonian) time $\mathrm{t}$ is extended to the deformed time $\mathrm{T}(\mathrm{t})=\mathrm{t}\left(1+\delta \times(\delta \mathrm{t} \uparrow)^{-\mathrm{T}(\mathrm{v}(\widetilde{\tau})}\right)=\mathrm{t}\left(1+\mathrm{t}^{-1} \times \tau^{-\mathrm{s}(\tau)}\right)$ with an $\mathrm{O}(1)$ directed multiplicative component for a $\tau=\delta \mathrm{t} \sim(1)$.

\subsection{Harmonic Oscillation}

Consider an orbit of the Harmonic oscillator

$$
\ddot{\mathrm{x}}+\mathrm{x}=0
$$


In $\mathrm{R}$ this equation is written as $\mathrm{X}^{\prime \prime}+\mathrm{X}=0$ where / denotes derivation with the deformed time $\mathrm{T}=\mathrm{t}\left(1+\mathrm{t}^{-1} \times(\tau)^{-\mathrm{s}(\mathrm{t})}\right)$, so that $\mathrm{dT} \approx \mathrm{dt}$, both for $\mathrm{t} \rightarrow 0$ or $\infty$. For $\mathrm{t}$ finite, $\mathrm{s}(\mathrm{t}) \approx 0$, but $\tau^{-\mathrm{s}}=\mathrm{O}(1)$ when $\mathrm{t} \rightarrow \infty$. Suppose the corresponding deformed orbit $\mathrm{X}$ is given by $\mathrm{X}=\mathrm{xe}^{\widetilde{\phi}(\eta, \mathrm{x}(\eta))}, \eta=\log \mathrm{T} / \mathrm{t}$. One verifies that nonlinear late time fluctuation $\phi(\mathrm{t})=\widetilde{\phi}(\eta, \mathrm{x}(\eta))$ satisfies the driven nonlinear equation

$$
\ddot{\phi}+(2 \dot{\mathrm{x}} / \mathrm{x}+\dot{\phi}) \dot{\phi}=-(\ddot{\mathrm{x}}+\mathrm{x}) / \mathrm{x} \text {. }
$$

when the explicit dependence of $\phi$ on $\mathrm{x}$ is disregarded, for simplicity (i.e., $\left|\phi_{\mathrm{x}}\right|<<\left|\phi_{\mathrm{t}}\right|$ etc). As a consequence, in a world admitting nontrivial small scale structures in the real number system, the classically sinusoidal orbits of an harmonic oscillator are expected to undergo a highly nonlinear late time evolution governed by (15) exposing an emergent interaction of slowly evolving nonlinear waves with (linear) sinusoidal wave. For finite values of $\mathrm{t}, \phi$ and its derivatives are negligible and we recover the pure harmonic oscillation. However, as $\mathrm{t} \rightarrow \infty$, small scale fluctuation in $\mathrm{t}$ is magnified, so that the scale invariant logarithmic variable $\eta \approx \tau^{-\mathrm{s}(\mathrm{t})} \approx\left(\mathrm{t}_{0} / \mathrm{t}\right)^{\mathrm{s}(\mathrm{t})}: \mathrm{O}(1)$, for a sufficiently large scale $\mathrm{t}_{0}=1 / \delta$ for the ordinary time. As a consequence, the original harmonic oscillation calibrated in ordinary time $t$ now is transferred to the new $\mathrm{O}(1)$ fluctuating motion in $\eta$ in which derivatives of $\phi$ ie, $\frac{\mathrm{d} \phi}{\mathrm{d} \eta}$ etc are non negligible, and the original linear oscillation would experience nonlinear perturbations.

Rewriting $\mathrm{Eq}(15)$ as

$$
\ddot{\mathrm{x}}+2 \dot{\phi} \dot{\mathrm{x}}+\left(1+\dot{\phi}^{2}\right) \mathrm{x}=-\ddot{\phi} \mathrm{x}
$$

We note that for a given deformation factor $\phi(\mathrm{t})$ original simple harmonic oscillation gets deformed into a driven Lienard type system (under the above simplifying assumption disregarding $\mathrm{x}$ dependence of $\phi$ ) because of the late time influence of the nonlinear internal time. For instance, choosing a deformation factor of the form $\dot{\phi}=\frac{\delta}{2}\left(\frac{1}{2} \mathrm{x}^{2}-1\right), 0<\delta<<1$ so that $\dot{\phi}^{2}$ term may be dropped, a late time nonlinear oscillation may be designed as the intrinsically generated Van der Pol equation

$$
\ddot{\mathrm{x}}+\delta\left(\mathrm{x}^{2}-1\right) \dot{\mathrm{x}}+\mathrm{x}=0(17)
$$

The closed circular phase paths of the harmonic oscillations would therefore be broken into families of phase paths spiraling towards the unique limit cycle of the Van der Pol equation Eq(17) in an asymptotically late time when the deformed nonlinear component of time $t$ is chosen suitably as above. For a small nonlinearity parameter $0<\delta<<1$, the unique limit cycle of the Van der Pol limit cycle has the exact amplitude (i.e. the value of $\mathrm{x}>0$ when $\dot{\mathrm{x}}=0) \mathrm{a}(\delta)=2$. According to the present scenario, as $\mathrm{t}$ approaches $1 / \delta$, all the harmonic oscillator orbits, excepting the one with amplitude 2 would open up and spiral toward the unique stable cycle (either inwardly or outwardly) in the asymptotically generated nonlinear system.

\subsection{Van der Pol Equation}

As pointed out already, the above derivation has a simple interpretation. A simple harmonic oscillator left undisturbed for an indefinite time would experience late time nonlinear oscillation because of the influence of hidden nonlinear structures which become activated at an asymptotic time. We gave a derivation of the Van der Pol oscillator like variations when the late time variation is modeled as a special amplitude variation for the original harmonic oscillator. Here we take the reversed problem. We begin with the Van der Pol oscillator equation

$$
\mathrm{x}^{\prime \prime}+\delta\left(\mathrm{x}^{2}-1\right) \mathrm{x}^{\prime}+\mathrm{x}=0
$$

and assume that explicit nonlinearity in the equation induces a transformation from the linear time variable $t$ to a nonlinear time $\tau=\phi(\delta \mathrm{t})$ with the condition that $\phi(\mathrm{t})$ is monotonic increasing and $\dot{\phi}(0)=1$ so that $\mathrm{d} \phi(\mathrm{t}) \approx \mathrm{dt}$ for $\mathrm{t} \approx 0$. This, in turn, implies that time derivatives (i.e. $\mathrm{x}^{\prime}$ and $\mathrm{x}^{\prime \prime}$ ) in the above equation are in fact with respect to $\tau$ rather than $\mathrm{t}$. As a consequence, re-expressing this equation in the ordinary time $\mathrm{t}$ we have 


$$
\frac{1}{\dot{\phi}^{2}} \ddot{\mathrm{x}}-\frac{\ddot{\phi}}{\dot{\phi}^{3}} \dot{\mathrm{x}}+\frac{1}{\dot{\phi}} \delta\left(\mathrm{x}^{2}-1\right) \dot{\mathrm{x}}+\mathrm{x}=0
$$

so that fixing $\phi$ by $\dot{\phi}(\mathrm{t})^{-1}=1-\delta \int\left(\mathrm{x}^{2}(\tau)-1\right) \mathrm{dt}$, we reproduce the harmonic oscillator equation for any $\delta>0$ when $0<\mathrm{t}<<1$, and we set $\dot{\phi}^{2} \approx 1$ (derivatives $\dot{\phi}$ etc are evaluated with the rescaled variable $\mathrm{t}$ ). With this choice of $\phi$ the two middle terms in the above equation cancel each other leading to the harmonic oscillator equation for a time scale arbitrarily close to 0 .

The advantage of this observation is the following. We now have a new non-perturbative method for computing solutions and estimating amplitude, frequency etc of the closed cycle of the Van der Pol equation. To briefly outline the actual procedure for computing the amplitude for the closed cycle of the Van der Pol oscillator, one begins with the harmonic oscillator solution $\mathrm{x}(\mathrm{t})=\mathrm{a} \sin \mathrm{t}$ for $\mathrm{t} \approx 0$. For a finite time $\mathrm{t}$, we replace $\mathrm{t}$ by the nonlinear time $\tau=\phi(\mathrm{t}):=\mathrm{t}+\mathrm{q}(\delta \mathrm{t})$, so that a solution of the nonlinear oscillator now is written as $\mathrm{x}(\tau)=\mathrm{a}(\mathrm{q}) \sin (\mathrm{t}+\mathrm{q}(\delta \mathrm{t}))$ where the functions $\phi(\mathrm{t})$ and the associated $\mathrm{q}(\delta \mathrm{t})$ are introduced as above. Consequently, $\mathrm{q}(0)=0$ and $\mathrm{q}(1)=1 \quad$ as $\mathrm{t}: 1 / \delta$. Writing $\mathrm{x}(\mathrm{t}+\mathrm{q})=\sum \mathrm{x}_{\mathrm{n}} \mathrm{q}^{\mathrm{n}}$ and $\mathrm{a}(\mathrm{q})=\sum \mathrm{a}_{\mathrm{n}} \mathrm{q}^{\mathrm{n}}$, and noting that the definition of $\mathrm{q}$ already involves $\mathrm{x}$, one can now develop an algorithm for calculating iteratively approximate solutions $\mathrm{x}_{\mathrm{n}}$ and the corresponding approximate amplitudes of the limit cycle. In general, the calculated phase trajectory need not represent a closed cycle, but as $t \rightarrow 1 / \delta$, all the trajectories of the Van der Pol system would be attracted toward the unique limit cycle, so the computed solution $\mathrm{x}(\tau)$, in the limit $\mathrm{q} \rightarrow 1^{-}$should correspond to the orbit of the limit cycle.

Details of the explicit computations will be taken up elsewhere. We close this Section dealing with the problem of transition of a linear system into a nonlinear mode and vice versa in the deformed real number system $R$ with the following remark:

In the present scenario, neighborhoods of $\mathrm{t}=0$ and $\mathrm{t}=\infty$ are disconnected sets. As a consequence, a linear harmonic oscillator in the $\mathrm{O}(1)$ linear time $\mathrm{t}$ in the connected segment of the form $\left(\delta, \delta^{-1}\right)$, will be transported into a nonlinear Van der Pol type nonlinear oscillator in an asymptotically distant connected line segment in the region $\mathrm{t}>\delta^{-1}$. On the other hand, a Van der Pol system in ordinary $\mathrm{O}(1)$ time variable $\mathrm{t}$ will be reduced into a linear harmonic oscillator in an infinitesimally small connected segment in $0<\mathrm{t}<\delta$. Although realized in a different setting, such a transition from a linear system to a nonlinear system (and vice versa) was also proposed in the homotopy analysis method of Liao [3] which seems to offer a much improved method for computing solution and relevant parameters in a nonlinear differential system. We shall explore the interesting problem of a homotopy interpretation of the above mentioned transition based on the deformed nonlinear time in future. The linear oscillator in the disconnected $\mathrm{O}(1)$ component $\left(\delta, \delta^{-1}\right)$ and a nonlinear oscillator in a disjoint component when $\mathrm{t}>\delta^{-1}$ as $\delta \rightarrow 0^{+}$can indeed be visualized as a homotopy transformation [3] on the (topological) space of differential operators.

\section{References}

[1] D.W. Jordan, P.Smith, Nonlinear Ordinary Differential Equations: An Introduction for scientists and Engineers (4th ed.), Oxford University Press (2007).

[2] Kevorkian. J, Cole.J. D., Multiple scale and Singular Perturbative Methods, springer, (1996).

[3] S. Liao, Homotopy Analysis Method in Nonlinear Differential Equations, Springer Berlin Heidelberg (2012).

[4] D.P. Datta and A.Ray Chaudhuri, Scale free analysis and prime number theorem, Fractals, 18,(2010),171-184.

[5] D.P.Datta and M.K.Bose, Higher derivative discontinuous solutions to linear ordinary differential equations: a new route to complexity? Chaos, Solitons and fractals,22,(2004),271-275.

[6] E.Nelson, International Set Theory: A New Approach to Nonstandard Analysis, Bulletin of the American Mathematical Society, Vol.83, Number 6, 1165-1198.

[7] T.Tao, Ultrafilters, Nonstandard analysis and epsilon management, http:// terry-tao.wordpress.com/2007/06/25.

[8] S.Raut and D.P.Datta, Analysis on a fractal set, Fractals, 17, (2009), 45-52; Erratum, ibid,17, (2009),547.

[9] S.Raut and D.P.Datta, Non-archimedean scale invariance and Cantor sets, Fractals, 18, (2010), 111-118.

[10] D.P.Datta, S.Raut and A.Ray Chaudhuri, Ultrametric Cantor sets and Growth of measure, P-adic Numbers, Ultrametric Analysis and Applications, Vol.3, No.1, (2011), 7-22.

[11] D.P.Datta, S.Raut and A.Ray Chaudhuri, Diffusion in a Class of fractal Sets, International journal of Applied Mathematics and Statistics, Vol.30; Issue No.6 (2012),34-50. 